\title{
CONSERVATION DES ÉCHANTILLONS DE LAIT (1)
}

\author{
par
}

\author{
E. G. VOIRET et BONAIMÉ \\ Directeur Chimiste \\ du Laboratoire Munieipal de la Ville de Lyon.
}

Cette conservation est de la plus haute importance pour le contrôle des laits. L'analyse a souvent lieu au bout d'un temps assez long après le prélèvement, d'une part ; d'autre part, les contreexpertises n'ont lieu, en général, que plusieurs mois après. L'analyse et les résultats ne sont comparables qu'autant que le lait n'est pas trop altéré. La formation d'une croûte de moisissures, en particulier, fixe une partie de la matière grasse qu'il est très difficile de réémulsionner. Ces faits sont surtout marqués, évidemment, en été et dans les pays chauds.

Le bichromate, conservateur le plus communément utilisé, est loin d'être sans inconvénient, du faît surtout de sa réduction assez rapide par les éléments du lait, du fait, également, que la couche de erème qui remonte à la surface n'en retient presque pas et se trouve à la merci de toutes les moisissures aérobies.

Le formol et le mélange formol trioxy-méthylène qui ont, par ailleurs, la faveur des autres, ne vont pas sans inconvénient, dont le principal est de tanner la caséine et de rendre celle-ci plus difficilement soluble au Gerber, et, bien que. des appareils émulseurs, comme celui décrit dans une note ci-jointe, permettent de rendre la dissolution facile grâce à la finesse des particules qu'ils réalisent, ces appareils sont trop peu répandus pour qu'il soit possible de les considérer comme un palliatif pratique.

Sur la proposition de M. BonaImé, nous avons expérimenté systématiquement, depuis un an, au Laboratoire municipal, le procédé simple suivant :

Il consiste à ajouter au lait, en mëme temps que le bichromate, $1 \%$ environ d'alcool amylique. Les résultats ont été parfaits et s'expliquent très bien pour les raisons suivantes :

$1^{\circ}$ L'alcool amylique est un bon antiseptique;

2 L'alcool amylique remontant à la surface avec la erème protège efficacement cette surface contre tout germe ou toute moisissure, tandis que le bichromate protège la couche aqueuse contre les ferments aérobies;

$3^{\circ}$ L'alcool amylique est un excellent agent d'émulsion. II diminue la tension superficielle de contact entre l'eau et la matière

(1) Annales des Falsiizations et des Fraudes, no 370-371-372, octobre-novembredécembre 1939, p. 404. 
grasse et, en même temps, limite, dans une large mesure, la tendance que peuvent avoir les grumeaux de caséine à s'agglomérer, de sorte que les échantillons, après plusieurs mois, sont non seulement intacts, mais peuvent facilement se réémulsionner, parfois par simple agitation, et ceci n'est pas un des moindres avantages.

Au total, l'association bichromate-alcool amylique réalise une protection totale de l'échantillon, chacun de ces deux produits étant insuffisants par lui-même et facilite énormément les opérations ultérieures.

L'alcool amylique est, par ailleurs, un produit non nocif, facile à manipuler, enfin sans action ni sur le lait lui-même ni sur la marche de l'analyse. Nous sommes convaincus que son emploi, surtout en période d'été ou dans les pays chauds, ne pourrait que donner des résultats satisfaisants.

Il serait pratique de l'utiliser soit, par exemple, sous forme d'ampoules de 1 à $2 \mathrm{~cm}^{3}$, soit avec un simple compte-gouttes.

\section{LA FABRICATION D'ACIDE LACTIQUE PUR}

par

\section{G. GENIN}

Ingénieur E. P. C.

Nous avons déjà à plusieurs reprises insisté sur l'intérêt que prend actuellement l'acide lactique en chimie industrielle et sur les procédés de fabrication de cet acide ou de ses sels. L'acide lactique est en effet aujourd'hui préparé industriellement par fermentation de divers hydrates de carbone, comme par exemple le sucre de maĩs, les mélasses ou le lactose contenu dans le sérum du lait. La solution aqueuse d'acide lactique que l'on obtient au cours de ces fermentations et des opérations qui les prolongent, contient certaines impuretés inorganiques ou organiques comme par exemple des sulfates de calcium et de sodium, des sucres non fermentés, des matières colorantes assez foncées et certaines substances azotées. Ces solutions d'acide lactique brut peuvent être employées dans certaines industries, comme par exemple pour la teinture des tissus ou le tannage du cuir, mais il est certaines autres applications qui exigent l'emploi d'un acide parfaitement pur.

Au cours de ces dernières années, en effet, des applications nouvelles importantes ont été trouvées pour l'acide lactique, à condition que le produit employé soit plus pur que l'acide généralement fabriqué jusqu'à présent. C'est ainsi, par exemple, qu'on a envisagé l'emploi de l'acide pur comme plastifiant et comme 